\title{
Differential mitochondrial DNA copy number in three mood states of bipolar disorder
}

\author{
Dong Wang ${ }^{1,4}$, Zongchang $\mathrm{Li}^{1,2}$, Weiqing Liu ${ }^{3}$, Jun Zhou ${ }^{1,4}$, Xiaoqian Ma ${ }^{1,4}$, Jinsong Tang ${ }^{1,4,5,6,7^{*}}$ \\ and Xiaogang Chen ${ }^{1,4,5,6,7^{*}}$
}

\begin{abstract}
Background: Accumulating evidences indicated that mitochondrial abnormalities were associated with bipolar disorder. As a sensitive index of mitochondrial function and biogenesis, Mitochondrial DNA copy number (mtDNAcn) may be involved in the pathophysiology of bipolar disorder.

Methods: Leukocyte relative mtDNAcn was measured by quantitative polymerase chain reaction in subjects with BD $(n=131)$ in manic, depressive, and euthymic symptoms. Thirty-four healthy individuals were used as comparison control. BD clinical symptomatology was evaluated by Young Mania Rating Scale (YMRS), Hamilton Depression Scale (HAM-D), Clinical Global Impression-Bipolar Disorder-Severity of Illness Scale (CGI-BD-S), and the Positive and Negative Syndrome Scale (PANSS).

Results: Compared to healthy controls, BD patients with manic and depressive symptoms presented significantly decreased mtDNAcn levels ( $p$-value $=0.009$ and 0.041, respectively). No significant differences were detected in mtDNAcn between euthymic patients and healthy controls. The mtDNAcn was negatively correlated with the number of relapses in manic patients $(\beta=-0.341, p=0.044)$.

Conclusions: Our study described the first evidence of (1) a significant decline of mtDNAcn in manic BD patients, (2) a similar decreased level of mtDNAcn between manic and depressed BD patients, (3) a negative correlation of mtDNAcn with number of relapses in patients suffering from manic states. Alterations of mtDNAcn in manic and depressed patients, which may reflect disturbances of energy metabolism, supported the role of mitochondrial abnormalities in the pathophysiology of BD.
\end{abstract}

Keywords: Bipolar disorder, Mitochondrial DNA copy number, Mania, Depression

\section{Background}

Bipolar disorder (BD) is a chronic and several psychiatric illness, characterized by recurrent episodes of mania and depression, and interspersed with remission period. Although many systems and pathways have been involved in the pathophysiology of $\mathrm{BD}$ together containing signal transduction pathways, various neurotransmitter abnormalities, mitochondrial and metabolic dysfunctions and so on [1]; the definitive mechanisms of BD remains to be fully elucidated.

\footnotetext{
* Correspondence: Tangjinsong@csu.edu.cn; Chenxiaogang@csu.edu.cn ${ }^{1}$ Department of Psychiatry, the Second Xiangya Hospital, Central South University, Changsha, Hunan, China

Full list of author information is available at the end of the article
}

Mitochondria serve as key organelles in eukaryotic cells, well known for synthesizing adenosine triphosphate (ATP) from glucose by oxidative phosphorylation for energy production. Especially, the energy source of neurons relies primarily on mitochondrial oxidative phosphorylation. Additionally, mitochondria also play a vital role in calcium signaling, cell resilience, apoptosis, the regulation of reactive oxygen species (ROS) and DNA damage [2-4]. Mitochondrial DNA (mtDNA) is a double-stranded DNA molecule with no introns and histones. Without protection of histones and due to limited DNA repair capacity, these mtDNA is very prone to oxidative or genotoxic injury. As a result, mitochondria may increase their own copy number to compensate for

(c) The Author(s). 2018 Open Access This article is distributed under the terms of the Creative Commons Attribution 4.0 International License (http://creativecommons.org/licenses/by/4.0/), which permits unrestricted use, distribution, and reproduction in any medium, provided you give appropriate credit to the original author(s) and the source, provide a link to the Creative Commons license, and indicate if changes were made. The Creative Commons Public Domain Dedication waiver (http://creativecommons.org/publicdomain/zero/1.0/) applies to the data made available in this article, unless otherwise stated. 
the defects or impairment. Excessive oxidative stresses in pathological conditions may induce structural and functional changes of mitochondrial [5]. Postmortem, brain imaging, genetic and peripheral cells studies all support that mitochondrial dysfunction is associated with BD [6-10]. Using magnetic resonance spectroscopy, some studies showed lower level of phosphocreatine, $\mathrm{N}$-acetyl-aspartate and inorganic phosphate and inorganic phosphate, which are supposed to reflect impaired mitochondrial function in several brain regions of living BD patients [6, 7]. There were lines of evidences revealing abnormal mitochondrial morphology in neurons of postmortem brain and peripheral cells of BD patients [8, 9]. Additionally, many SNPs across the genome of mitochondria were associated with $\mathrm{BD}[10,11]$. These observed changes maybe indicate an important role of mitochondria in the pathogenesis of BD.

Mitochondrial DNA copy number (mtDNAcn) that reflects the mitochondrial function and biogenesis could be measured per cell [12]. Abnormal mtDNAcn has been suggested to associate with various psychiatric disorders [13-15]. With regard to $\mathrm{BD}$, few studies were conducted to address the possible role of mtDNAcn in BD. Vawter et al. firstly found the alteration of mtDNAcn in a post-mortem study [16], but other three post-mortem studies got negative results [17-19]. In peripheral blood leukocytes, Chang et al. revealed that euthymic BD patients had a lower mtDNAcn than control group [20]; While de Sousa et al. found no significant differences in mtDNA content between depressed BD patients and health controls [21]. Furthermore, the mtDNA content still did not have a significant decrease after lithium carbonate treatment.

Mitochondrial dysfunction may be a cause of $\mathrm{BD}$ symptoms. Given the heterogeneity of the disease itself and the inconsistent results from studies, it is necessary to find more evidence to interpret the effect of mitochondrial dysfunction in BD. Human leukocytes have been proved to reflect virtually the plasma oxidative damage to DNA [22]. Heretofore, no studies have assessed the mtDNA content in leukocytes of patients suffering mania or different states of BD. In this study, mtDNAcn was measured in BD patients with three different states and healthy individuals to identify how mtDNA content is altered in different mood states. We hypothesized that mtDNAcn variation may associate with $\mathrm{BD}$, and would differentiate mood states as well as bipolar euthymia from healthy controls.

\section{Methods}

\section{Participants}

The study was approved by the ethics committee of the Second Xiangya Hospital of Central South University. All subjects voluntarily participated in the research and signed the informed consent. These procedures were performed in accordance with Declaration of the Helsinki. Acute BD patients were recruited from the inpatient and outpatient departments of the Second Xiangya Hospital in Hunan Province of China. The Euthymic patients of BD were primarily recruited from the outpatient clinic.

BD type I is defined by a pattern of depressive episodes and manic episodes. Subjects with BD type I were diagnosed by two or more trained psychiatrists using the Structured Clinical Interview for DSM- IV Axis I Disorders (SCID-IV) and its researcher version with psychotic screen (SCID-I/P). The exclusion criteria for BD patients included electroconvulsive therapy within past 3 months; substance abuse; other mental illness; neurological diseases; serious medical conditions; positive pregnancy test or lactation. Meanwhile, healthy volunteers were recruited by advertisements from local communities. Healthy controls with substance use, history of mental or neurological disorders, serious medical disorders, positive pregnancy test or lactation were excluded from the study (see Additional file 1).

\section{Assessment}

One hundred thirty-one subjects with BD type I and 34 healthy volunteers were enrolled in the study. There were 55 patients meeting the Diagnostic and Statistical Manual of Mental Disorders Fourth Edition (DSM-IV) criteria suffering from manic episodes, 47 patients in depressive episodes and 29 patients in euthymic states. Young Mania Rating Scale (YMRS), Hamilton Depression Scale (HAM-D), Clinical Global Impression-Bipolar Disorder-Severity of Illness Scale (CGI-BD-S) were used to assess the symptom severity respectively. Euthymic $\mathrm{BD}$ patients were clinically stable for more than 6 months and the index score for CGI-BD-S $\leq 2$. When all patients were recruited in the study, the psychiatric assessment and blood collection would be completed in next $24 \mathrm{~h}$.

\section{Assays}

Blood samples $(3 \mathrm{ml})$ were collected in EDTA tubes from the 165 participants and kept at $-80{ }^{\circ} \mathrm{C}$. Genomic DNA was extracted from $200 \mu \mathrm{l}$ blood samples following the manufacturer's protocol using QIAamp DNA Mini Kit (Qiagen, Hilden, Germany). The quantity and purity of the DNA were measured by Nanodrop 2000 spectrophotometer.

The detailed methods and procedures were described in our previous research [13]. In brief, the mtDNAcn was evaluated by the ratio of the amount of mtDNAcn (ND1) to a reference nuclear single copy gene (hemoglobin subunit beta HBB) copy number. The primers for ND1 were as follow: $\mathrm{F}$ (5-CCCTAAAACCCGCCACATCT-3) and $\mathrm{R}$ (5-GAGCGATGGTGAGAGCTAAGGT-3). The primers for (HBB) were $\mathrm{F}$ (5-GTGCACCTGACTCCTGAGGAGA-3) and R (5-CCTTGATACCA ACCTGCCCAG-3). 
The assays were conducted by using LightCycler ${ }^{\circ} 480$ SYBR Green I Master on a Roche LightCycler 480 ma- $^{\circ}$ chine (Roche, Manheim, Germany). The double-standard curves method of relative quantification PCR was employed, which is an improved, simple, accurate method [23]. The quantitative assay was based on amplification using conditions, primer sequences according to Xing et al. [24]. PCR cycling condition of ND1 gene was $95^{\circ} \mathrm{C}$ 10 min for 1 cycle, 30 cycles of $95{ }^{\circ} \mathrm{C}$ for $15 \mathrm{~s}, 60^{\circ} \mathrm{C}$ for $1 \mathrm{~m}$. The cycling condition of $\mathrm{HBB}$ gene was $95{ }^{\circ} \mathrm{C}$ $10 \mathrm{~min}, 40$ cycles of $95^{\circ} \mathrm{C} 15 \mathrm{~s}, 56^{\circ} \mathrm{C}$ for $1 \mathrm{~m}$. Each sample was run in triplicate using $10 \mathrm{ng}$ DNA templates in a 10 ul reaction volume and randomly assigned to 96-well plates. The ND1 and HBB genes PCR reactions were performed on separate plates with the same samples located at the same positions., The measures of mtDNAcn for each sample were obtained by the ratio of ND1 copy number to HBB copy number from standard curves. The ratio was normalized to a calibrator DNA in order to standardize between different independent assays. The calibrator DNA sample was from a health control individual and used for comparison with results of different runs. The calibrator DNA sample was diluted 1:4 to build a five-point standard curve between 0.0195 and $20 \mathrm{ng} / \mathrm{ul}$. The amplification efficiencies of the standards were between 92.3 and $108.2 \%$. A negative control and a calibrator DNA were enrolled in each run. The inter-assay coefficient of variation $(\mathrm{CV})$ was $0.15-3.5 \%$, and the intra-assay CV was $1.13-4.37 \%$.

\section{Statistical analysis}

Statistical analysis was conducted using Minitab 16 (Minitab Inc., Pennsylvania) and SPSS 23.0 (SPSS Inc., Chicago, IL) for windows. Dichotomous categorical variables in groups were compared using Chi-square test. Continuous variables with normal distribution were analyzed using $\mathrm{T}$ test or one-way analysis of variance analysis (ANOVA) test. The mtDNAcn in four groups were skewed distributed, and then adapted into normal distribution using Box-Cox transformation in Minitab software [25]. The Box-Cox (1964) transformation model in Minitab could select the best mathematical function to obtain a normal distribution of the transformed data [26]. The primary outcome analysis was conducted using ANOVA test. When significance was shown in ANOVA, Generalized linear model and Bonferroni post hoc test were performed to examine the intergroup differences of mtDNAcn, after adjusting sexuality, age, years of education, body mass index (BMI). Effect sizes were calculated using Cohen's eta squared $\eta^{2}$ in ANOVA test and $R^{2}$ in multiple regression analyses [27]. Cohen J suggested effect sizes $\eta^{2}$ of $<0.01$ were small, 0.06 medium, and $\geq 0.14$ large. For each $\mathrm{BD}$ group, multiple regression analyses were used to explore the association between mtDNAcn and other variables. Descriptive statistics were shown as the mean \pm standard deviation or median (interquartile range), and significance level was set at $P<0.05$ (two-tailed).

\section{Results}

Demographic and clinical data of the sample

Table 1 lists demographic, clinical and medication information, and the mtDNAcn median (range) of the study sample. BD patients and healthy controls did not differ in terms of age, gender, BMI, months of education (all $P>0.05$ ). Second generation antipsychotic drugs were prescribed for $\mathrm{BD}$ patients in the research, and their doses were converted to $\mathrm{CPZ}$ equivalents available [28]. As showed, there were no significant differences in using CPZ equivalents, anticonvulsants, benzodiazepines and Lithium (all $P>0.05$ ). Manic subjects demonstrated more severe symptoms than depressive and euthymic subjects on Young Mania Rating Scale; and depressed group showed significantly higher HDRS scores than manic and euthymic groups. To identify effect of illness history on mtDNAcn, the detailed information about duration of illness and number of previous episodes were recorded. Both Length of illness and number of previous episodes were similar in three groups $(P>0.05)$.

\section{MtDNA copy number in subjects with BD patients and controls}

After transformation, the mean mtDNAcn of four groups were $0.5 \pm 0.40$ (manic group), $0.57 \pm 0.44$ (depressed group), $0.86 \pm 0.49$ (euthymic group), $0.73 \pm 0.45$ (healthy control). The results of ANOVA showed a significant difference $(F=$ 4.78, $\left.\mathrm{df}=3161, P=0.003, \eta^{2}=0.085\right)$. After controlling for age, gender, BMI, years of schooling as covariates, there remained a significant difference $(F=2.54, \mathrm{df}=6158, P=$ $0.023, \eta^{2}=0.087$ ). Besides, we found on the model, these covariates did not associate with mtDNAcn (all $P>0.05$ ). The Bonferroni post hoc test revealed that, comparing with healthy group, depressed and manic groups showed a significantly lower level of mtDNAcn respectively, $(P=$ $\left.0.041, \eta^{2}=0.068, \quad \mathrm{~s}=0.083 ; \quad P=0.009, \eta^{2}=0.049, \quad s=0.081\right)$ (Fig. 1). No differences were detected in the mtDNAcn between manic group and depressed group $(P>0.05)$. The euthymic patients had a similar level of mtDNAcn with healthy volunteers $(P>0.05)$ (Table 2). Although the mtDNAcn of BD euthymic group were increased in comparison with manic and depressed BD group, the differences did not reach a significant level (euthymic vs. manic $P=0.056$, euthymic vs. depressive $P=0.11$ ).

\section{Association of mtDNAcn with clinical characteristics within bipolar subjects}

Multiple regression analysis was conducted in each group to confirm the relationship between mtDNAcn and clinical information (Table 2). For the manic group, the mtDNAcn was negatively correlated with the 
Table 1 Demographic, clinical and medication information of bipolar patients and healthy controls

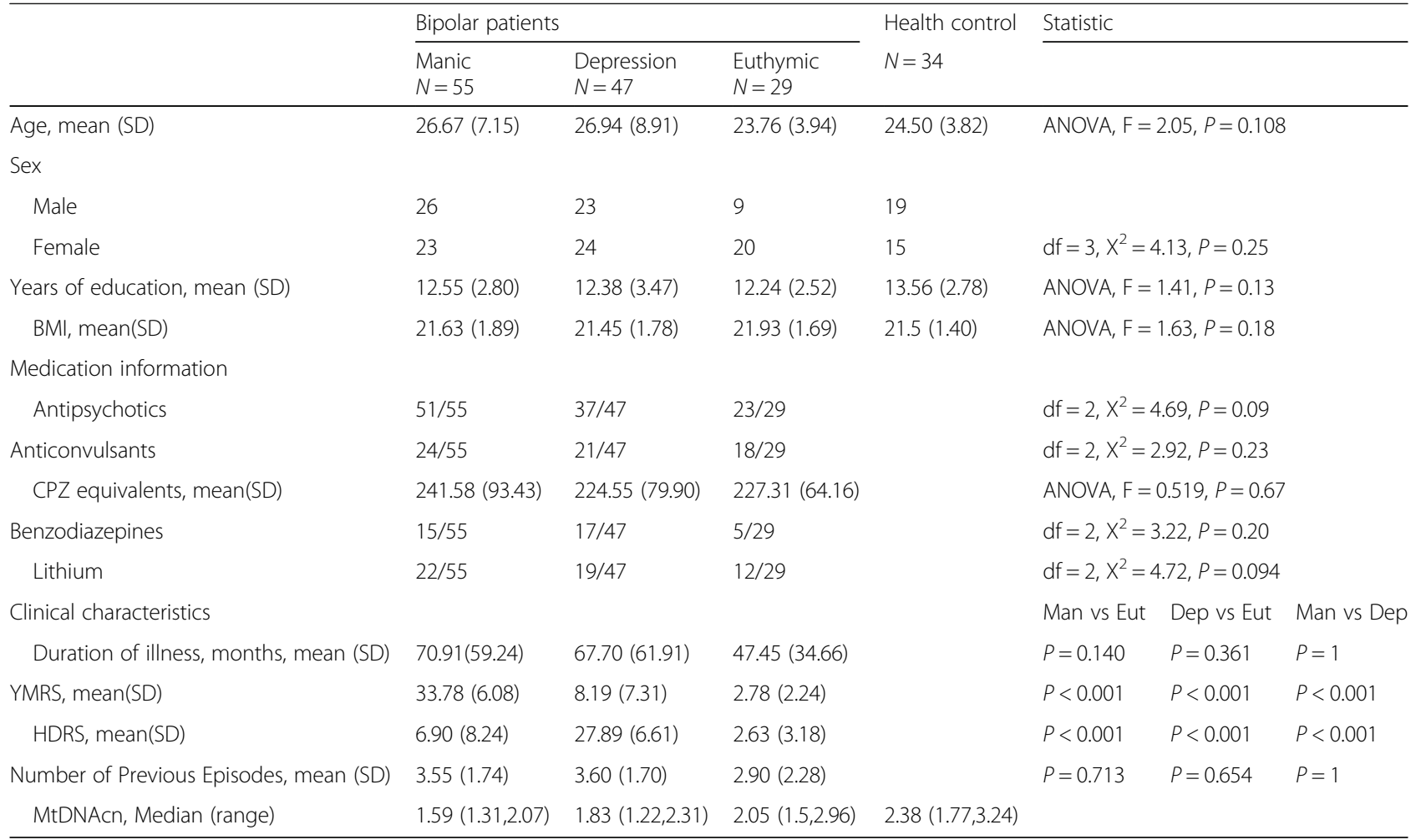

Abbreviations: $B M I$ body mass index, $S D$ standard deviation, YMRS young mania rating scale. HDRS hamilton depression rating scale, ANOVA one-way analysis of variance, Man manic, Dep depression, Eut euthymic

number of relapses $(\beta=-0.341, t=-2.61, P=0.044, s$ $=0.039$ ) (Fig. 2), while no similar findings happened in depressed group $(\beta=0.319, t=1.19, P=0.964, s=$ $0.044)$. The other clinical characteristics or variables had no significant association with mtDNAcn in BD patients (all $P>0.05$ ).

\section{Discussion}

To our knowledge, this is the first study to examine mtDNA content across the depressed, manic and euthymic states in $\mathrm{BD}$ patients. This current research has three main findings. (1) The acute BD patients had markedly lower mtDNA amount than healthy controls,

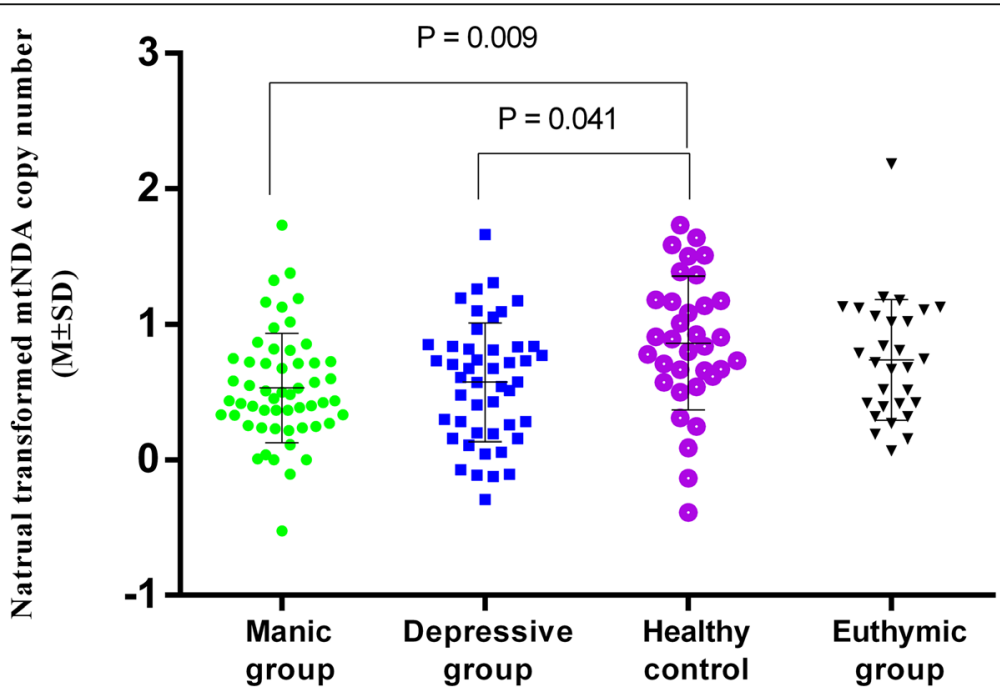

Fig. 1 MtDNA copy number in manic, depressive, euthymic BD patients and healthy controls 
Table 2 Effect sizes for healthy control versus patients groups and for multiple regression analyses in each patients group

\begin{tabular}{|c|c|c|c|c|c|c|}
\hline & \multicolumn{2}{|l|}{ Manic group } & \multicolumn{2}{|c|}{ Depressive group } & \multicolumn{2}{|c|}{ Euthymic group } \\
\hline & $\overline{\mathrm{ES}(\mathrm{SE})}$ & $P$ & $\overline{E S}$ & $P$ & $\overline{E S}$ & $P$ \\
\hline healthy control & $0.068^{\mathrm{C}}(0.081)$ & $0.009^{\mathrm{a} *}$ & $0.049^{\complement}(0.083)$ & $0.041^{a_{*}}$ & $0.008^{\mathrm{C}}(0.091)$ & $1^{\mathrm{a}}$ \\
\hline \multicolumn{7}{|l|}{ Clinical characteristics } \\
\hline Duration of illness & $0.002^{\mathrm{d}}(0.001)$ & $0.825^{b}$ & $0.003^{\mathrm{d}}(0.001)$ & $0.743^{b}$ & $0.009^{d}(0.004)$ & $0.77^{\mathrm{b}}$ \\
\hline YMRS/HDRS & $0.040^{d}(0.012)$ & $0.262^{b}$ & $0.100^{d}(0.016)$ & $0.796^{\mathrm{b}}$ & $0.259^{d}(0.076)$ & $0.212^{\mathrm{t}}$ \\
\hline Number of Previous Episodes & $0.116^{d}(0.039)$ & $0.044^{\mathrm{b} *}$ & $0.102^{d}(0.044)$ & $0.964^{\mathrm{b}}$ & $0.006^{d}(0.065)$ & $0.679^{k}$ \\
\hline CGI-BD-S & $0.009^{d}(0.067)$ & $0.632^{b}$ & $0.02^{d}(0.088)$ & $0.724^{\mathrm{b}}$ & $0.072^{d}(0.081)$ & $0.536^{\mathrm{b}}$ \\
\hline
\end{tabular}

ES effect size, SE standard error, YMRS young mania rating scale, HDRS hamilton depression rating scale, CGI-BD-S clinical global impression-bipolar disorderseverity of illness scale, ${ }^{\text {adjjusted }} p$-values of one way ANOVA test; ${ }^{b}$ adjusted $p$-values of multiple regression analyses; ${ }^{C}$ Cohen's eta squared $\eta^{2}$ in ANOVA test; ${ }^{d}{ }^{2}$ in multiple regression analyses; ${ }^{*} P<0.05$

after controlling covariates; there were no differences in mtDNAcn between manic patients and depressed patients. (2) The euthymic group showed a comparable content of mtDNA with healthy control group. (3) The mtDNAcn was inversely correlated with the number of relapses in BD subjects during manic episodes.

Change of mtDNAcn is an important sensitive index of mitochondrial dysfunction and oxidative stress [29]. Our findings indicated that there may be explicit mitochondrial impairments during episodes of BD. In the current study, we firstly reported decreased mtDNA amount in peripheral leukocytes of BD patients undergoing manic episodes. Our data suggested, manic group may display a similar level of mtDNA with depressive group. In contrast, there was nearly normal level of mtDNA in euthymic patients. Hence, we speculate that, leukocyte mtDNA content may change with mood states in BD. Nicod et al. suggested the mtDNA content of patients changed with time during 8 -week treatment in major depression [30]. Acute patients could recover after treatment, and gradually transit to euthymic states. According to the findings and clinical observations above, the mtDNAcn of acute patients in peripheral blood might be gradually restored to some extent as time goes on.

The results of prior data on mtDNAcn in peripheral leukocytes and brain tissues of mood disorder were not consistent. Compatible with our result, de Sousa et al. showed a slight decreased mtDNAcn in depressive patients with BD type I, although the differences just failed to be significant $(P=0.05)$, due to a small sample $(N=7)$ [21]. Chang et al. described that mtDNAcn was still significantly lower in the BD euthymic patients [20]. One plausible explanation is that mtDNAcn was negatively correlated with age in BD [21], and their subjects were older than ours. Accumulated evidences demonstrate BD may be an accelerated aging disease [31]. Aging was also reported to be associated with the downregulation of mtDNA-associated genes and a decline level of mtDNAcn [32]. Therefor accelerated aging in BD may affect the levels of mtDNA. According to threshold hypothesis of mtDNAcn control [12], low mtDNAcn triggers the upregulation of mtDNA replication and high mtDNAcn triggers the machinery to degradate mtDNA. Taken together, low threshold and accelerated aging may

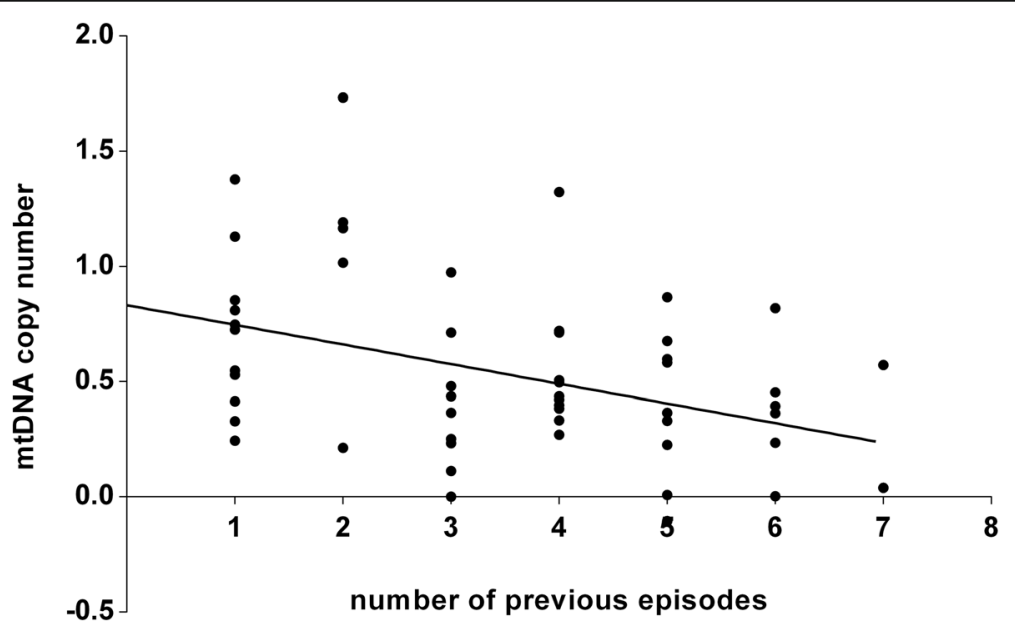

Fig. 2 Correlation between number of previous episodes and mtDNA copy number $(\beta=-0.341, t=-2.61, p=0.044)$ within manic BD patients 
induce the decrease of mtDNAcn. Regarding mtDNAcn in postmortem brains, three out of four studies reported a negative result in BD [16-19]. It was not clear about what kinds of mood states that those subjects suffered from. Thus, the comparison cannot be made with ours. Additional, another innegligible factor is using different tissues or cells in studies, which may confound the results. .

MtDNA polymerase gamma (POLG) is the only one polymerase responsible for the mtDNA replication. POLG is prone to oxidative damage,and its deleterious variants were suggested as a risk for BD [33]. Munkholm et al. revealed downregulation of POLG expression in BD across mania, hypomania, depression or mixed states in peripheral leukocyte [34]. We suppose that POLG downregulation in acute phases of BD may be another important point of resulting in decrease of mtDNAcn.

There are also several evidences for abnormal mtDNA amounts in major depression [35-38]. A large sample multicenter study reported patients with major depression showed increased mtDNA level than controls in saliva and blood [37]. Two studies found a lower peripheral mtDNA level in both depressive and euthymic states $[35,38]$. Nicod et al. suggested mtDNA content of patients changed with time in major depression [30].Overall it is not consistent about changes of mtDNA content in major depression and bipolar depression. However all studies indicate the importance of mtDNA in mood disorder. Future research is warranted to explore the relationship in different states of mood disorders.

The relationship observed between mtDNAcn and the number of episodes in manic patients deserves attention. In the current study, we found that mtDNA content was negatively correlated with the number of episodes in $\mathrm{BD}$ patients. BD patients often experience recurrent episodes or relapses which appear to cause chronicity of this illness and vulnerability for high stress response [39]. The subjects suffering manic episode are at a high stress level [40]. The "sensitivity" state of oxidative stress may accelerate illness course, and cause cell deficits as well as rapid decline of mitochondrial function.. However, similar findings were not found in the depressive subjects of this study. Hence, the results should be interpreted cautiously, due to the heterogeneity and complexity of BD.

Although antipsychotic agents and mood stabilizers were reported to exert some effect on mitochondrial function $[4,41-43]$. de Sousa and our previous studies showed that six or eight weeks lithium or risperidone treatment have no significant effect on mtDNAcn in subjects with psychiatric disorders [13, 21]. Accordingly, we suppose the mtDNAcn of the acute patients would make no significant changes after short-term treatment. Whereas our euthymic patients were medically stabilized for more than 6 months, and may have enough time to recover from various damage in cell.
There are several limitations to be considered. Since we didn't have more clinical data, including smoking status, glucose intolerance, and serum lipid, the possible influence of these variables cannot be completely excluded. Although using peripheral blood is more convenient and less invasive to quantify $\mathrm{mtDNAcn}$, these finding cannot be extrapolated mechanically to other tissues. In addition, only mtDNAcn was measured in this cross-sectional data, it does not indicate any information about causal relationship between oxidative stress, mtDNA variations, and $\mathrm{BD}$. More biological parameters and longitudinal studies regarding mitochondria involved in the pathophysiology of $\mathrm{BD}$ are warranted in future.

\section{Conclusion}

This study provides novel observations that Leukocyte mtDNA content was significantly decreased in BD patients suffering manic and depressive episodes, as compared with healthy controls. Our results also showed mtDNAcn was negatively correlated with the number of relapses in manic BD patients. Mitochondrial abnormalities in acute patients might involve in the pathophysiology of $\mathrm{BD}$. The findings maybe promote a further understanding on the role of mitochondria in $\mathrm{BD}$. Although our data indicated the mtDNAcn might change with mood states, the underlying mechanisms are complex and still unclear. More effort for a better explanation of the processes engaged in BD is required in further investigation.

\section{Additional file}

Additional file 1: Excluded neurological diseases and other serious physical conditions in the study. (DOCX $15 \mathrm{~kb}$ )

\section{Abbreviations}

ANCOVA: One-way analysis of covariance; ANOVA: one-way analysis of variance; ATP: adenosine triphosphate; BD: Bipolar disorder; BMI: Body Mass Index; CGI-BD-S: Clinical Global Impression-Bipolar Disorder-Severity of IIIness Scale; CPZ: chlorpromazine; Dep: Depression; DSM-IV: Diagnostic and Statistical Manual of Mental Disorders Fourth Edition; Eut: Euthymic; HAMD: Hamilton Depression Scale; Man: Manic; mtDNAcn: mitochondrial DNA; mtDNAcn: Mitochondrial DNA copy number; POLG: polymerase gamma; ROS: reactive oxygen species; SCID-IV: Structured Clinical Interview for DSMIV Axis I Disorders; SD: Standard Deviation; YMRS: Young Mania Rating Scale

\footnotetext{
Funding

This work was supported by the social development program of Wuxi [CSE31N1724 to D.W]; National Natural Science Foundation of China [81471361 to X.C, 81371480 to J.T], Human Provincial Innovation Foundation for Postgraduate [12700-150140005 to Z.L]. The funders had no role in study design, data collection and analysis, decision to publish or preparation of the manuscript
}

Availability of data and materials

Data set is not publicly available for the informed consent. However, data could be available from the corresponding author on reasonable request. 


\section{Authors' contributions}

XC, JT, designed the study. DW, ZL, JZ, XM performed the data collection and WL, DW performed data analyses. DW was responsible for manuscript writing. All authors have read and approved the final manuscript.

\section{Ethics approval and consent to participate}

The study was approved by the ethics committee of the Second Xiangya Hospital of Central South University. All subjects voluntarily participated in the research and signed the informed consent.

\section{Competing interests}

The authors declare that they have no competing interests.

\section{Publisher's Note}

Springer Nature remains neutral with regard to jurisdictional claims in published maps and institutional affiliations.

\section{Author details}

'Department of Psychiatry, the Second Xiangya Hospital, Central South University, Changsha, Hunan, China. ${ }^{2}$ Laboratory of Medical Genetics, School of Life Sciences, Central South University, Changsha, Hunan, China. ${ }^{3}$ Department of Psychiatry, First Affiliated Hospital of Kunming Medical University, Kunming, Yunnan, China. ${ }^{4}$ Mental Health Institute, the Second Xiangya Hospital, Central South University, Changsha, Hunan, China. ${ }^{5}$ National Clinical Research Center on Mental Disorders, Changsha, Hunan, China. ${ }^{6}$ National Technology Institute on Mental Disorders, Changsha, Hunan, China. ${ }^{7}$ Hunan Key Laboratory of Psychiatry and Mental Health, Changsha, Hunan, China.

\section{Received: 5 October 2017 Accepted: 2 May 2018}

\section{Published online: 25 May 2018}

\section{References}

1. Sigitova E, Fisar Z, Hroudova J, Cikankova T, Raboch J. Biological hypotheses and biomarkers of bipolar disorder. Psychiatry Clin Neurosci. 2017;71(2):77-103.

2. Bolisetty S, Jaimes EA. Mitochondria and reactive oxygen species: physiology and pathophysiology. Int J Mol Sci. 2013;14(3):6306-44

3. Niizuma K, Yoshioka H, Chen H, Kim GS, Jung JE, Katsu M, Okami N, Chan $\mathrm{PH}$. Mitochondrial and apoptotic neuronal death signaling pathways in cerebral ischemia. Biochim Biophys Acta. 2010;1802(1):92-9.

4. Scaini G, Rezin GT, Carvalho AF, Streck EL, Berk M, Quevedo J. Mitochondrial dysfunction in bipolar disorder: evidence, pathophysiology and translational implications. Neurosci Biobehav Rev. 2016;68:694-713.

5. Clay HB, Sillivan S, Konradi C. Mitochondrial dysfunction and pathology in bipolar disorder and schizophrenia. Int J Dev Neurosci. 2011;29(3):311-24.

6. Bertolino A, Frye M, Callicott JH, Mattay VS, Rakow R, Shelton-Repella J, Post R, Weinberger DR. Neuronal pathology in the hippocampal area of patients with bipolar disorder: a study with proton magnetic resonance spectroscopic imaging. Biol Psychiatry. 2003;53(10):906-13.

7. Sikoglu EM, Jensen JE, Vitaliano G, Liso NAA, Renshaw PF, Frazier JA, Moore CM. Bioenergetic measurements in children with bipolar disorder: a pilot 31P magnetic resonance spectroscopy study. PLoS One. 2013;8(1):e54536.

8. Cataldo AM, MCPhie DL, Lange NT, Punzell S, Elmiligy S, Ye NZ, Froimowitz $M P$, Hassinger LC, Menesale EB, Sargent LW, et al. Abnormalities in mitochondrial structure in cells from patients with bipolar disorder. Am J Pathol. 2010;177(2):575-85.

9. Kvajo M, Dhilla A, Swor DE, Karayiorgou M, Gogos JA. Evidence implicating the candidate schizophrenia/bipolar disorder susceptibility gene G72 in mitochondrial function. Mol Psychiatry. 2008:13(7):685-96.

10. Ueno H, Nishigaki Y, Kong QP, Fuku N, Kojima S, Iwata N, Ozaki N, Tanaka M. Analysis of mitochondrial DNA variants in Japanese patients with schizophrenia. Mitochondrion. 2009;9(6):385-93.

11. Muller DJ, Zai CC, Shinkai T, Strauss J, Kennedy JL. Association between the DAOA/G72 gene and bipolar disorder and meta-analyses in bipolar disorder and schizophrenia. Bipolar Disord. 2011;13(2):198-207.

12. Clay MLL, Deng JJ, Bai Y. Number matters: control of mammalian mitochondrial DNA copy number. J Genet Genomics. 2009;36(3):125-31.

13. Li Z, Hu M, Zong X, He Y, Wang D, Dai L, Dong M, Zhou J, Cao H, Lv L, et al. Association of telomere length and mitochondrial DNA copy number with risperidone treatment response in first-episode antipsychotic-naive schizophrenia. Sci Rep. 2015;5:18553.
14. Bersani FS, Morley C, Lindqvist D, Epel ES, Picard M, Yehuda R, Flory J, Bierer LM, Makotkine I, Abu-Amara D, et al. Mitochondrial DNA copy number is reduced in male combat veterans with PTSD. Prog Neuro-Psychopharmacol Biol Psychiatry. 2016;64:10-7.

15. Yoo HJ, Park M, Kim SA. Difference in mitochondrial DNA copy number in peripheral blood cells between probands with autism spectrum disorders and their unaffected siblings. World J Biol Psychiatry. 2017; 18(2):151-6.

16. Vawter MP, Tomita H, Meng F, Bolstad B, Li J, Evans S, Choudary P, Atz M, Shao L, Neal C, et al. Mitochondrial-related gene expression changes are sensitive to agonal-pH state: implications for brain disorders. Mol Psychiatry. 2006;11(7):615. 663-679

17. Torrell H, Montana E, Abasolo N, Roig B, Gaviria AM, Vilella E, Martorell L. Mitochondrial DNA (mtDNA) in brain samples from patients with major psychiatric disorders: gene expression profiles, mtDNA content and presence of the mtDNA common deletion. Am J Med Genet B Neuropsychiatr Genet. 2013;162B(2):213-23.

18. Sabunciyan S, Kirches E, Krause G, Bogerts B, Mawrin C, Llenos IC, Weis S. Quantification of total mitochondrial DNA and mitochondrial common deletion in the frontal cortex of patients with schizophrenia and bipolar disorder. J Neural Transn (Vienna). 2007;114(5):665-74.

19. Kakiuchi C, Ishiwata M, Kametani M, Nelson C, Iwamoto K, Kato T. Quantitative analysis of mitochondrial DNA deletions in the brains of patients with bipolar disorder and schizophrenia. Int J Neuropsychopharmacol. 2005;8(4):515-22.

20. Chang CC, Jou SH, Lin TT, Liu CS. Mitochondrial DNA variation and increased oxidative damage in euthymic patients with bipolar disorder. Psychiatry Clin Neurosci. 2014;68(7):551-7.

21. de Sousa RT, Uno M, Zanetti MV, Shinjo SM, Busatto GF, Gattaz WF, Marie SK, Machado-Vieira R. Leukocyte mitochondrial DNA copy number in bipolar disorder. Prog Neuro-Psychopharmacol Biol Psychiatry. 2014;48:32-5.

22. Liu CS, Tsai CS, Kuo CL, Chen HW, Lii CK, Ma YS, Wei YH. Oxidative stressrelated alteration of the copy number of mitochondrial DNA in human leukocytes. Free Radic Res. 2003;37(12):1307-17.

23. Zhang C, Xu S, Huang X. A novel and convenient relative quantitative method of fluorescence real time RT-PCR assay based on slope of standard curve. Prog Biochem Biophys. 2005;32(9):883-8. (http://europepmc.org/ abstract/cba/596524)

24. Xing J, Chen M, Wood CG, Lin J, Spitz MR, Ma J, Amos Cl, Shields PG, Benowitz NL, Gu J. Mitochondrial DNA content: its genetic heritability and association with renal cell carcinoma. J Natl Cancer Inst. 2008:100(15):1104.

25. Box GEP, Cox DR. An analysis of transformations. J R Stat Soc Ser B Methodol. 1964;26(2):211-52. http://www.jstor.org/stable/2984418.

26. Sakia RM. The box-cox transformation technique: a review. J R Stat Soc. 1992;41(2):169-78. ( http://www.jstor.org/stable/2348250)

27. Cohen J. Statistical power analysis for the behavioral sciences. 2nd ed. Hillsdale, N.J: L. Erlbaum Associates; 1988.

28. Woods SW. Chlorpromazine equivalent doses for the newer atypical antipsychotics. J Clin Psychiatry. 2003;64(6):663-7.

29. Malik AN, Czajka A. Is mitochondrial DNA content a potential biomarker of mitochondrial dysfunction? Mitochondrion. 2013;13(5):481-92.

30. Nicod J, Wagner S, Vonberg F, Bhomra A, Schlicht KF, Tadic A, Mott R, Lieb $K$, Flint J. The amount of mitochondrial DNA in blood reflects the course of a depressive episode. Biol Psychiatry. 2016;80(6):e41.

31. Rizzo LB, Costa LG, Mansur RB, Swardfager W, Belangero SI, Grassi-Oliveira R, McIntyre RS, Bauer ME, Brietzke E. The theory of bipolar disorder as an illness of accelerated aging: implications for clinical care and research. Neurosci Biobehav Rev. 2014:42:157-69.

32. Hartmann N, Reichwald K, Wittig I, Drose S, Schmeisser S, Luck C, Hahn C, Graf M, Gausmann U, Terzibasi E, et al. Mitochondrial DNA copy number and function decrease with age in the short-lived fish Nothobranchius furzeri. Aging Cell. 2011;10(5):824-31.

33. Kasahara T, Ishiwata M, Kakiuchi C, Fuke S, Iwata N, Ozaki N, Kunugi H, Minabe $Y$, Nakamura K, Iwata Y, et al. Enrichment of deleterious variants of mitochondrial DNA polymerase gene (POLG1) in bipolar disorder. Psychiatry Clin Neurosci. 2017;71(8):518-29.

34. Munkholm K, Peijs L, Vinberg M, Kessing LV. A composite peripheral blood gene expression measure as a potential diagnostic biomarker in bipolar disorder. Transl Psychiatry. 2015;5:e614.

35. Chang CC, Jou SH, Lin TT, Lai TJ, Liu CS. Mitochondria DNA change and oxidative damage in clinically stable patients with major depressive disorder. PLoS One. 2015;10(5):e0125855. 
36. He Y, Tang J, Li Z, Li H, Liao Y, Tang Y, Tan L, Chen J, Xia K, Chen X. Leukocyte mitochondrial DNA copy number in blood is not associated with major depressive disorder in young adults. PLoS One. 2014;9(5):e96869.

37. Cai N, Chang S, Li Y, Li Q, Hu J, Liang J, Song L, Kretzschmar W, Gan X, Nicod J, et al. Molecular signatures of major depression. Curr Biol. 2015; 25(9):1146-56.

38. Kageyama Y, Kasahara T, Kato M, Sakai S, Deguchi Y, Tani M, Kuroda K, Hattori K, Yoshida S, Goto Y, et al. The relationship between circulating mitochondrial DNA and inflammatory cytokines in patients with major depression. J Affect Disord. 2018;233:15-20.

39. Pfaffenseller B, Fries GR, Wollenhaupt-Aguiar B, Colpo GD, Stertz L, Panizzutti B, Magalhaes PV, Kapczinski F. Neurotrophins, inflammation and oxidative stress as illness activity biomarkers in bipolar disorder. Expert Rev Neurother. 2013;13(7):827-42.

40. Kapczinski F, Dal-Pizzol F, Teixeira AL, Magalhaes PV, Kauer-Sant'Anna M, Klamt F, Pasquali MA, Quevedo J, Gama CS, Post R. A systemic toxicity index developed to assess peripheral changes in mood episodes. Mol Psychiatry. 2010;15(8):784-6.

41. Inuwa IM, Peet M, Williams MA. QSAR modeling and transmission electron microscopy stereology of altered mitochondrial ultrastructure of white blood cells in patients diagnosed as schizophrenic and treated with antipsychotic drugs. Biotech Histochem. 2005;80(3-4):133-7.

42. Bachmann RF, Wang Y, Yuan P, Zhou R, Li X, Alesci S, Du J, Manji HK. Common effects of lithium and valproate on mitochondrial functions: protection against methamphetamine-induced mitochondrial damage. Int J Neuropsychopharmacol. 2009:12(6):805-22.

43. Eftekhari A, Azarmi Y, Parvizpur A, Eghbal MA. Involvement of oxidative stress and mitochondrial/lysosomal cross-talk in olanzapine cytotoxicity in freshly isolated rat hepatocytes. Xenobiotica. 2016;46(4):369-78.

\section{Ready to submit your research? Choose BMC and benefit from}

- fast, convenient online submission

- thorough peer review by experienced researchers in your field

- rapid publication on acceptance

- support for research data, including large and complex data types

- gold Open Access which fosters wider collaboration and increased citations

- maximum visibility for your research: over $100 \mathrm{M}$ website views per year

At BMC, research is always in progress.

Learn more biomedcentral.com/submissions 